\title{
LAGRANGIAN MINIMAL SURFACES IN KÄHLER-EINSTEIN SURFACES OF NEGATIVE SCALAR CURVATURE
}

\author{
YNG-ING LEE
}

Assume $M_{1}, M_{2}$ are closed surfaces with hyperbolic metrics $g_{1}, g_{2}$ respectively. Then $\left(M_{1}, g_{1}\right) \times\left(M_{2}, g_{2}\right)$ is a Kähler-Einstein surface of negative scalar curvature. In this paper we prove the following results:

Theorem 1. Let $\sum$ be a closed surface with $\chi\left(\sum\right)=P_{1} \chi\left(M_{1}\right)=P_{2} \chi\left(M_{2}\right)$, where $P_{1}, P_{2}$ are positive integers. Suppose

$$
f=\left(f_{1}, f_{2}\right): \sum \rightarrow\left(M_{1}, g_{1}\right) \times\left(M_{2}, g_{2}\right),
$$

where $\operatorname{deg} f_{1}=P_{1}, \operatorname{deg} f_{2}=-P_{2}$ or $\operatorname{deg} f_{1}=-P_{1}, \operatorname{deg} f_{2}=P_{2}$. Then there exists a unique minimal surface in the homotopy class of $f$ and this minimal surface is Lagrangian.

Remark. The case $M_{1}=M_{2}$ and $P_{1}=P_{2}=1$ is proved by R. Schoen in [15].

Theorem 2. Suppose $f=\left(f_{1}, f_{2}\right): T^{2} \rightarrow\left(M_{1}, g_{1}\right) \times\left(M_{2}, g_{2}\right)$, where $T^{2}$ is a torus and $\left[f\left(T^{2}\right)\right]=\alpha_{1} \times \alpha_{2}, \alpha_{i} \in H_{1}\left(M_{i}\right), i=1,2$. Then there exists a unique minimal surface in the homotopy class of $f$ and this minimal surface is Lagrangian.

Theorem 1 and 2 can also be interpreted as the existence and uniqueness of Lagrangian minimal surfaces in the depicted homotopy classes and consequently these Lagrangian minimal surfaces are area minimizing in their homtopy classes. We will discuss more about this point in next section. Along

The research was partially supported by an Alfred P. Sloan Doctoral Disseration Fellowships and NSF grants DMS-9100383 in IAS. 
the line of this second intepretation we study the relations between symplectic geometry and Riemannian geometry, and show the following result.

Proposition 1. Let $(N, g)$ be a Kähler-Einstein surface with $c_{1}<0$, where $c_{1}$ is the first Chern class of $(N, g)$. Suppose $\beta \in H_{2}(N)$ and there exists an embedded totally real surface $\sum_{r}$ of genus $r$ representing $\beta$. Then every connected embedded minimal surface in $\beta$ has genus no less than $r$. The equality holds if and only if the embedded minimal surface is Lagrangian.

Remark 1. The Kähler-Einstein surface $N$ in Proposition 1 is not necessarily a product space.

Remark 2. The complex structure with respect to which $\sum_{r}$ is totally real may be different from the one of $g$. All we need is that the first Chern classes of two complex structures are the same.

The paper is organized as follows. In Section 1 we give an introduction of the known interesting results about the Lagrangian minimal surfaces. In Section 2 we review some necessary background of harmonic map theory. In the last section we proves the theorems.

Acknowledgement. This work is based on part of the author's thesis. She would like to express her sincere thanks and deep gradititude to her advisor, professor Richard M. Schoen. She also like to thank the referee for some valued suggestions on the revision of the original manuscript.

\section{INTRODUCTION}

A symplectic manifold $\left(N^{2 n}, \omega\right)$ is a $2 n$-dimensional manifold with a closed nondegenerate 2 -form $\omega$, which is called a symplectic form. The most natural and important submanifolds in a symplectic manifold are the so-called Lagrangian submanifolds. They are n-dimensional submanifolds of $\left(N^{2 n}, \omega\right)$ on which $\omega$ vanishes. A Kähler manifold can also be viewed as a symplectic manifold with its Kähler form as the symplectic form. One can then combine the Riemannian structure and symplectic structure and study the Lagrangian minimal submanifolds which are both Lagrangian and minimal. 
Lagrangian minimal surfaces have some very nice features. For instance, every Lagrangian minimal surface in $R^{4}$ or a K-3 surface is holomorphic with respect to some compatible complex structure (see [8], [21]). Therefore, in these two cases Lagrangian minimal surfaces have all the same properties as holomorphic curves. Although this does not hold in general, there are still other interesting properties about Lagrangian minimal surfaces.

When $N$ is a Kähler manifold of nonpositive Ricci curvature, every Lagrangian minimal submanifold in $N$ is stable. (See [3], [12], [13].) The uniqueness in our Theorem 1 depends heavily on this property. Moreover, J. G. Wolfson has shown that a totally real minimal surface in a Kähler-Einstein surface with $c_{1}<0$ is Lagrangian [21]. It indicates that the Lagrangian minimal surfaces in Kähler-Einstein surfaces with $c_{1}<0$ have a kind of "rigidity". These two features make it possible to develop a deformation theory for Lagrangian minimal surfaces in the moduli space of Kähler-Einstein metrics with $c_{1}<0$ [11]. We will discuss this in another paper.

R. Harvey and B. Lawson studied the so-called special Lagrangian submanifolds in $R^{2 n}$, which are calibrated by the $n$-form $R e\left(d z_{1} \wedge \cdots \wedge d z_{n}\right)$. (See [7],[8].) Being calibrated implies volume minimizing. So in particular, the special Lagrangian submanifolds are Lagrangian minimal submanifolds. Using the idea of calibrations, one can show that every Lagrangian minimal submanifold in a Kähler-Einstein manifold $N$ with $c_{1}(N)=0$ is volume minimizing. It is false for the case $c_{1}=\lambda \omega$ with $\lambda>0$, and is unknown for the case $c_{1}=\lambda \omega$ with $\lambda<0$. The uniqueness of the Lagrangian minimal surfaces in the classes mentioned in Theorem 1 and 2 implies that these Lagrangian minimal surfaces are volume minimizing in their homotopy classes. This gives some positive evidence for the case $c_{1}=\lambda \omega, \lambda<0$.

Unfortunately, very few is known about the existence of Lagrangian minimal submanifolds except those examples constructed by R. Harvey and B. Lawson in [8] and some very obvious ones. In fact, R. Bryant proved that both $c_{1}$ (represented by the Ricci form) and $\omega$ vanish on Lagrangian minimal submanifolds [2], therefore one cannot expect the existence of Lagrangian minimal submanifolds in a Kähler manifold in general. However, this obstruction disappears if we restrict the discussion on a Kähler-Einstein manifold. R. Bryant showed 
the local existence of Lagrangian minimal submanifolds in this situation [2]. In Theorem 1 and 2, we obtain the global existence for some cases.

\section{Preliminaries}

Most of the techniques we use are from the theory of harmonic maps. So in this section we want to introduce some basic definitions and facts about harmonic maps.

Let $M, N$ be compact manifolds with Riemannian metrics $g, h$ respectively. Given a smooth map $u: M \rightarrow N$, one can define the energy of $u$ by

$$
E(u)=\int_{M} \operatorname{Tr}_{g} u^{*}(h) d \mu_{g},
$$

where $\operatorname{Tr}_{g}$ means trace with respect to the metric $g$ and $d \mu_{g}$ denotes the volume measure associated to the metric $g$. The critical points of this energy function are called harmonic maps. If $N$ is isometrically embedded in $R^{k}$, the harmonic map equation can be written as

$$
\Delta u^{i}+g^{\alpha \beta} \Gamma_{j l}^{i}(u(x)) \frac{\partial u^{j}}{\partial x^{\alpha}} \frac{\partial u^{l}}{\partial x^{\beta}} \equiv 0, \quad i=1, \ldots, k .
$$

Here the double indices means a summation and $\Gamma_{j l}^{i}$ are the Christoffel symbols of $h$.

If $\operatorname{dim} M=2$ and $z=x^{1}+i x^{2}$ is a complex coordiate on $M$, denote

$$
\frac{\partial}{\partial z}=\frac{1}{2}\left(\frac{\partial}{\partial x^{1}}-i \frac{\partial}{\partial x^{2}}\right), \quad \frac{\partial}{\partial \bar{z}}=\frac{1}{2}\left(\frac{\partial}{\partial x^{1}}+i \frac{\partial}{\partial x^{2}}\right) .
$$

One can define the Hopf differential of $u$ on $M$ :

$$
\begin{aligned}
\Phi_{u} & =u^{*}(h)^{2,0} d z^{2} \\
& =h_{i j} \frac{\partial u^{i}}{\partial z} \frac{\partial u^{j}}{\partial z} d z^{2} \\
& =\frac{1}{4}\left(\left|\frac{\partial u}{\partial x^{1}}\right|^{2}-\left|\frac{\partial u}{\partial x^{2}}\right|^{2}-2 i<\frac{\partial u}{\partial x^{1}}, \frac{\partial u}{\partial x^{2}}>\right) d z^{2} .
\end{aligned}
$$

It can be checked by $(*)$ that if $u$ is a harmonic map then the Hopf differential is a holomorphic quadratic differential on $M$. The map $u$ is conformal if and only if $\Phi_{u} \equiv 0$.

Now we also assume $\operatorname{dim} N=2$. Let $z=x^{1}+i x^{2}, u=u^{1}+i u^{2}$ be complex coordinates on $M, N$ respectively. Then the metrics of $M, N$ are of 
the forms $\lambda^{2}(z)|d z|^{2}, \sigma^{2}(u)|d u|^{2}$ respectively. The map $u$ is represented locally by $u=u(z)$. Then the harmonic map equation becomes

$$
\frac{\partial^{2} u}{\partial z \partial \bar{z}}+2 \frac{\partial \log \sigma}{\partial u} \frac{\partial u}{\partial z} \frac{\partial u}{\partial \bar{z}}=0
$$

and the Hopf differential $\Phi_{u}$ can be expressed as

$$
\Phi_{u}=\sigma^{2}(u)\left(\frac{\partial u^{1}}{\partial z} \frac{\partial u^{1}}{\partial z}+\frac{\partial u^{2}}{\partial z} \frac{\partial u^{2}}{\partial z}\right) d z^{2}=\sigma^{2}(u) \frac{\partial u}{\partial z} \frac{\partial \bar{u}}{\partial z} d z^{2} .
$$

Define

$$
|\partial u|^{2}(z)=\lambda^{-2}(z) \sigma^{2}(u(z))\left|\frac{\partial u}{\partial z}\right|^{2}, \quad|\bar{\partial} u|^{2}(z)=\lambda^{-2}(z) \sigma^{2}(u(z))\left|\frac{\partial u}{\partial \bar{z}}\right|^{2} .
$$

From the harmonic equation $(* *)$ and a standard argument [1], it follows that if $|\partial u|^{2}$ (respectively $|\bar{\partial} u|^{2}$ ) is not identically zero, it has at most a discrete set of zeros. Again by $(* *)$, one can derive the following Bochner equations

$$
\begin{aligned}
& \Delta_{g} \log |\partial u|^{2}=-2 K_{h} \cdot J(u)+2 K_{g} \\
& \Delta_{g} \log |\bar{\partial} u|^{2}=2 K_{h} \cdot J(u)+2 K_{g},
\end{aligned}
$$

at points where $|\partial u|^{2}$ (respectively $|\bar{\partial} u|^{2}$ ) is nonzero [17]. Here $J(u)=|\partial u|^{2}-$ $|\bar{\partial} u|^{2}$ is the Jacobian determinant of $u$, and $K_{g}, K_{h}$ are the Gaussian curvature fuctions of $g, h$ respectively. Using the same argument as in [17], their Theorem 3.1 can be generalized to the following lemma:

Lemma 1. Suppose $\left(M_{1}, g_{1}\right),\left(M_{2}, g_{2}\right)$ are closed Riemann surfaces of genus $r_{1}, r_{2}$ respectively, the Gaussian curvature $K_{g_{2}} \leq 0$ and $f:\left(M_{1}, g_{1}\right) \rightarrow\left(M_{2}, g_{2}\right)$ is a harmonic map of degree $s \neq 0$. We have

(a) If $r_{1}=r_{2} \geq 1$ and $|s|=1$, then $f$ is a diffeomorphism.

(b) If $r_{1}, r_{2} \geq 1$ and $\left|\chi\left(M_{1}\right)\right|=\left|s \cdot \chi\left(M_{2}\right)\right|$, where $\chi$ is the Euler characteristic number, then $f: M_{1} \rightarrow M_{2}$ is a covering map with $J(f) \neq 0$.

Proof. Integrate both sides of equations (1), (2) and follow a standard residue argument, we get

$$
\sum_{p \in M_{1}} n_{p}=-s\left(4 r_{2}-4\right)+\left(4 r_{1}-4\right) \quad \text { provided }|\partial f| \not \equiv 0 \text { on } M_{1}
$$

$$
\sum_{p \in M_{1}} m_{p}=s\left(4 r_{2}-4\right)+\left(4 r_{1}-4\right) \quad \text { provided }|\bar{\partial} f| \not \equiv 0 \text { on } M_{1}
$$


where $n_{p}$ (respectively $m_{p}$ ) is the order of zero of $\partial f$ (respectively $\bar{\partial} f$ ) at $p$.

Suppose $s>0$, it follows that $|\partial f|^{2}$ is not identically zero. Because we have $\chi\left(M_{1}\right)=s \cdot \chi\left(M_{2}\right)$, the equality (3) becomes $\sum_{p \in M_{1}} n_{p}=0$. That is, $n_{p}=0$ for any $p \in M_{1}$. Therefore, $|\partial f|^{2}>0$ on $M_{1}$. We first claim that $J(f) \geq 0$ on $M_{1}$. Suppose to the contrary that

$$
D=\left\{p \in M_{1}: J(f)(p)<0\right\}
$$

is not empty. Then

$$
|\partial f|^{2}<|\bar{\partial} f|^{2} \text { on } D \text { and }|\partial f|^{2}=|\bar{\partial} f|^{2} \text { on } \partial D \text {. }
$$

Because $|\partial f|^{2}>0$ on $M_{1}$, it follows that $|\bar{\partial} f|^{2}>0$ on $D \cup \partial D$. We can substract (2) from (1) to obtain

$$
\Delta \log \frac{|\partial f|^{2}}{|\bar{\partial} f|^{2}}=-4 K_{g_{2}} J(f) \text { on } D \cup \partial D
$$

Thus $\log \frac{|\partial f|^{2}}{|\bar{\partial} f|^{2}}$ is smooth and superharmonic on $D$. Also

$$
\log \frac{|\partial f|^{2}}{|\bar{\partial} f|^{2}} \leq 0 \text { on } D \text { and } \log \frac{|\partial f|^{2}}{|\bar{\partial} f|^{2}}=0 \text { on } \partial D \text {. }
$$

By the minimum principle it follows that

$$
\log \frac{|\partial f|^{2}}{|\bar{\partial} f|^{2}} \equiv 0 \text { on } D \text {, i.e. } J(f) \equiv 0 \text { on } D .
$$

It is a contradiction. Thus $J(f) \geq 0$ on $M_{1}$.

Because $J(f) \geq 0$ on $M_{1}$ and it is not identically zero, we then have $J(f)(p)=0$ if and only if $|\partial f(p)|=|\bar{\partial} f(p)|=0$ as in Proposition 2.2 of [17]. Suppose to the contrary that $J(f)(p)=0$ but $|\partial f(p)|=|\bar{\partial} f(p)|>0$. Taking a small disk $V$ about $p$ where $|\bar{\partial} f|>0$, from (1) and (2) one has

$\Delta \log \frac{|\partial f|^{2}}{|\bar{\partial} f|^{2}}=-4 K_{g_{2}}\left(|\partial f|^{2}-|\bar{\partial} f|^{2}\right) \leq-\left.4 K_{g_{2}} \operatorname{Bar} \partial f\right|^{2}\left(\frac{|\partial f|^{2}}{|\bar{\partial} f|^{2}}-1\right) \leq c \log \frac{|\partial f|^{2}}{|\bar{\partial} f|^{2}}$,

where $c$ is a positive constant. That is, the non-negative function $h=\log \frac{|\partial f|^{2}}{|\bar{\partial} f|^{2}}$ satisfies $\Delta h \leq c h$. By Lemma $6^{\prime}$ of [10], it implies $\int_{V} h d A \leq c^{\prime} h(p)$ for some constant $c^{\prime}>0$. Thus $h \equiv 0$ in $V$, which is a contradiction. Hence

$$
|\partial f(p)|=|\bar{\partial} f(p)|=0
$$


Because of $|\partial f|^{2}>0$ on $M_{1}$, we have $J(f)>0$ on $M_{1}$ and $f$ is a covering map (it is a diffeomorphism when $s=1$ ). If $s<0$, a similar argument shows that the same result also holds.

\section{MAIN THEOREMS}

Let $N$ be a compact Kähler surface. The necessary and sufficient conditions for $\beta \in H_{2}(N)$ to have a representative which is a Lagrangian surface are $[\omega](\beta)=0$ and $c_{1}(N)(\beta)=0$. (See [4], [11].) These two conditions in a KählerEinstein surface become only one condition $[\omega](\beta)=0$. A class satisfying the condition is called a Lagrangian class. It only makes sense to discuss the existence of Lagrangian minimal surfaces and whether a surface is Lagrangian in a Lagrangian class. Here we first determine the Lagrangian classes in $N=$ $\left(M_{1}, g_{1}\right) \times\left(M_{2}, g_{2}\right)$. We have the formula

$$
H_{2}(N)=H_{2}\left(M_{1}\right) \times H_{0}\left(M_{2}\right)+H_{0}\left(M_{1}\right) \times H_{2}\left(M_{2}\right)+H_{1}\left(M_{1}\right) \times H_{1}\left(M_{2}\right) .
$$

The Kähler form $\omega$ in the product metric is $\omega=\omega_{1}+\omega_{2}$, where $\omega_{i}$ is the Kähler form and is also the volume form for $\left(M_{i}, g_{i}\right), i=1$ or 2 . It is clear that the action of $\omega$ on $\alpha_{1} \times \alpha_{2}$ is 0 , where $\alpha_{i} \in H_{1}\left(M_{i}\right), i=1,2$. Thus $\alpha_{1} \times \alpha_{2}$ is a Lagrangian class. The action of $\omega$ on $\left[M_{1} \times 1\right]$ is

$$
\int_{\left[M_{1} \times 1\right]} \omega=\int_{M_{1}} \omega_{1}=-2 \pi \chi\left(M_{1}\right)
$$

where $\chi\left(M_{1}\right)$ is the Euler number of $M_{1}$. The last equality follows from GaussBonnet Theorem and the facts that $g_{1}$ has constant curvature -1 and $\omega_{1}$ is the volume form on $M_{1}$. Similarly, we have

$$
\int_{\left[1 \times M_{2}\right]} \omega=-2 \pi \chi\left(M_{2}\right) \text {. }
$$

Thus $P_{1} \cdot\left[M_{1} \times 1\right]-P_{2} \cdot\left[1 \times M_{2}\right]$ is a Lagrangian class, if $P_{1} \chi\left(M_{1}\right)=P_{2} \chi\left(M_{2}\right)$ and $P_{1}, P_{2}$ are positive integers. Hence a general Lagrangian homology class in $\left(M_{1}, g_{1}\right) \times\left(M_{2}, g_{2}\right)$ can be written as a linear combination of the classes $P_{1} \cdot\left[M_{1} \times 1\right]-P_{2} \cdot\left[1 \times M_{2}\right]$ and $\alpha_{1} \times \alpha_{2}$, where $\alpha_{1} \in H_{1}\left(M_{1}\right)$ and $\alpha_{2} \in H_{1}\left(M_{2}\right)$.

In some of the Lagrangian homotopy classes, one can prove very strong results. 
Theorem 1. Let $\sum$ be a closed surface with $\chi\left(\sum\right)=P_{1} \chi\left(M_{1}\right)=P_{2} \chi\left(M_{2}\right)$, where $P_{1}, P_{2}$ are positive integers. Suppose

$$
f=\left(f_{1}, f_{2}\right): \sum \rightarrow\left(M_{1}, g_{1}\right) \times\left(M_{2}, g_{2}\right),
$$

where $\operatorname{deg} f_{1}=P_{1}, \operatorname{deg} f_{2}=-P_{2}$ or $\operatorname{deg} f_{1}=-P_{1}, \operatorname{deg} f_{2}=P_{2}$. Then there exists a unique minimal surface in the homotopy class of $f$ and this minimal surface is Lagrangian.

The proof depends on the next lemma, which is a generalization of Lemma 2.10 in [15].

Lemma 2. Suppose $M, M_{1}, M_{2}$ are closed Riemann surfaces and $f_{1}, f_{2}$ are harmonic maps from $(M, g)$ onto $\left(M_{1}, g_{1}\right),\left(M_{2}, g_{2}\right)$ respectively. Moreover, $J\left(f_{1}\right)>0, J\left(f_{2}\right)<0$ and $K_{g_{1}}=K_{g_{2}}=-1$. If the absolute values of their Hopf differentials coincide, i.e. $\left|\Phi_{f_{1}}\right|=\left|\Phi_{f_{2}}\right|$, then

$$
\left|\partial f_{1}\right|=\left|\bar{\partial} f_{2}\right| \text { and }\left|\bar{\partial} f_{1}\right|=\left|\partial f_{2}\right| .
$$

Proof. Substract (2) from (1), we get

$$
\Delta \log \frac{\left|\partial f_{1}\right|^{2}}{\left|\bar{\partial} f_{1}\right|^{2}}=-4 K_{g_{1}} J\left(f_{1}\right)=4\left(\left|\partial f_{1}\right|^{2}-\left|\bar{\partial} f_{1}\right|^{2}\right) .
$$

If we define $w_{1}=\log \frac{\left|\partial f_{1}\right|}{\left|\bar{\partial} f_{1}\right|}$, then

$$
2 \Delta w_{1}=4\left|\partial f_{1}\right|\left|\bar{\partial} f_{1}\right|\left(\frac{\left|\partial f_{1}\right|}{\left|\bar{\partial} f_{1}\right|}-\frac{\left|\bar{\partial} f_{1}\right|}{\left|\partial f_{1}\right|}\right)=4\left|\Phi_{f_{1}}\right|\left(e^{w_{1}}-e^{-w_{1}}\right) .
$$

That is,

$$
\Delta w_{1}=4\left|\Phi_{f_{1}}\right| \sinh w_{1} .
$$

Similarly for $w_{2}=\log \frac{\left|\partial f_{2}\right|}{\left|\bar{\partial} f_{2}\right|}$, we have

$$
\Delta w_{2}=4\left|\Phi_{f_{2}}\right| \sinh w_{2}
$$

Let $n_{p}$ (respectively $m_{p}, n_{p}^{\prime}$ and $m_{p}^{\prime}$ ) be the order of zero of $\partial f_{1}$ (respectively $\bar{\partial} f_{1}, \partial f_{2}$ and $\left.\bar{\partial} f_{2}\right)$ at $p$. Because

$$
\left|\Phi_{f_{1}}\right|=\left|\Phi_{f_{2}}\right| \quad \text { i.e. } \quad\left|\partial f_{1}\right|\left|\bar{\partial} f_{1}\right|=\left|\partial f_{2}\right|\left|\bar{\partial} f_{2}\right|,
$$


one has $n_{p}+m_{p}=n_{p}^{\prime}+m_{p}^{\prime}$. On the other hand, $J\left(f_{1}\right)>0$ and $J\left(f_{2}\right)<0$ (therefore $n_{p}=0, m_{p}^{\prime}=0$ ), it follows that $n_{p}=m_{p}^{\prime}$ and $m_{p}=n_{p}^{\prime}$. Hence $w_{1}+w_{2}$ is a regular function on $M$ satisfying

$$
\Delta\left(w_{1}+w_{2}\right)=4|\Phi|\left(\sinh w_{1}+\sinh w_{2}\right),
$$

where $|\Phi|=\left|\Phi_{f_{1}}\right|=\left|\Phi_{f_{2}}\right|$. At a maximal point $p$ of $w_{1}+w_{2}$ one has

$$
\Delta\left(w_{1}+w_{2}\right)(p) \leq 0
$$

it follows that

$$
\sinh w_{1}(p)+\sinh w_{2}(p) \leq 0 .
$$

Hence

$$
\left(w_{1}+w_{2}\right)(p) \leq 0 .
$$

However, at a minimal point $q$ of $w_{1}+w_{2}$ one has

$$
\left(w_{1}+w_{2}\right)(q) \geq 0 \text {. }
$$

Therefore

$$
w_{1}+w_{2} \equiv 0 \quad \text { i.e. } \quad \frac{\left|\partial f_{1}\right|}{\left|\bar{\partial} f_{1}\right|} \frac{\left|\partial f_{2}\right|}{\left|\bar{\partial} f_{2}\right|}=1 .
$$

This together with the fact that $\left|\partial f_{1}\right|\left|\bar{\partial} f_{1}\right|=\left|\partial f_{2}\right|\left|\bar{\partial} f_{2}\right|$ follows that

$$
\left|\bar{\partial} f_{1}\right|=\left|\partial f_{2}\right| \text { and }\left|\partial f_{1}\right|=\left|\bar{\partial} f_{2}\right| \text {. }
$$

We also need the following existence result by R. Schoen and S. T. Yau.

Lemma 3 ([16]). Let $N$ be a compact Riemannian manifold. Let $f$ be a continuous map from a Riemann surface $M$ into $N$. Suppose the induced map of $f$ on the fundamental group given by $f_{*}: \pi_{1}(M) \rightarrow \pi_{1}(N)$ is injective; then there is a branched minimal immersion $h: M \rightarrow N$ so that $h_{*}=f_{*}$ on $\pi_{1}(M)$ and the induced area of $h$ is least among all maps with the same action on $\pi_{1}(M)$. If $\pi_{2}(N)=0$, then $h$ can indeed be deformed from $f$ continously.

Proof of Theorem 1. Assume $\operatorname{deg} f_{1}=P_{1}, \operatorname{deg} f_{2}=-P_{2}$. We first prove that there exists at most one minimal surface in the homotopy class of $f$. For any hyperbolic metric $g^{\prime}$ on $\sum$, there exists a unique harmonic map $f_{g^{\prime}}$ homotopic to $f$ from $\left(\sum, g^{\prime}\right) \rightarrow\left(M_{1}, g_{1}\right) \times\left(M_{2}, g_{2}\right)$. (See [6], [9].) Denote the Teichmüller space of $\sum$ by $\mathcal{T}_{r}$, where $r>1$ is the genus of $\sum$. Let $P$ be a point in $\mathcal{T}_{r}$ 
and $\left(\sum, g^{\prime}\right)$ be a representative for $P$. Because of the conformal invariance of the energy function $E(\cdot)$, we can define $E(P)=E\left(f_{g^{\prime}}\right)$ whose value is independent of the metric used to represent $P$. The function $E$ is proper on $\mathcal{T}_{r}$ and its critical points are precisely the minimal immersions homotopic to $f$ from $\sum \rightarrow N$. (See [16].) We claim that the minimal surfaces in the homotopy class, if there exists any, are in fact Lagrangian. Assume $\left(\sum, g\right)$ is a critical point. Then $f_{g}:\left(\sum, g\right) \rightarrow\left(M_{1}, g_{1}\right) \times\left(M_{2}, g_{2}\right)$ is a conformal harmonic map [18], and the projection maps into the factors satisfy:

$f_{g}^{(1)}:\left(\sum, g\right) \rightarrow\left(M_{1}, g_{1}\right)$ is a harmonic map of degree $P_{1}$, and

$f_{g}^{(2)}:\left(\sum, g\right) \rightarrow\left(M_{2}, g_{2}\right)$ is a harmonic map of degree $-P_{2}$.

Because $\chi\left(\sum\right)=P_{1} \chi\left(M_{1}\right)=P_{2} \chi\left(M_{2}\right)$, by Lemma 1 we get that $f_{g}^{(1)}, f_{g}^{(2)}$ are covering maps and $J\left(f_{g}^{(1)}\right)>0, J\left(f_{g}^{(2)}\right)<0$. The conformality of $f_{g}$ simply says that $\Phi_{f_{g}^{(1)}}=-\Phi_{f_{g}^{(2)}}$, and hence from Lemma 2 we have $\left|\partial f_{g}^{(1)}\right|=\left|\bar{\partial} f_{g}^{(2)}\right|$ and $\left|\bar{\partial} f_{g}^{(1)}\right|=\left|\partial f_{g}^{(2)}\right|$. Therefore, $J\left(f_{g}^{(1)}\right)=-J\left(f_{g}^{(2)}\right)$ and we conclude that the symplectic form $\omega$ vanishes on $f_{g}\left(\sum\right)$. That is, $f_{g}\left(\sum\right)$ is Lagrangian. Therefore, we conclude that every minimal surface in this homotopy class is a Lagrangian minimal immersion. (The immersion follows from the fact that $J\left(f_{g}^{(1)}\right)>0$.) Lagrangian minimal immersions in a Kähler manifold of nonpositive Ricci curvature are always stable. (See [3], [12], [13].) In particular, the result holds on a Kähler-Einstein surface of negative scalar curvature. Because $E$ is proper on $\mathcal{T}_{r}$ and all of its critical points are local minima, we then conclude by elementary Morse theory that there is at most one critical point.

For any given metric $g^{\prime}$, there exists a unique harmonic map $f_{g^{\prime}}$ homotopic to $f$ from $\left(\sum, g^{\prime}\right) \rightarrow\left(M_{1}, g_{1}\right) \times\left(M_{2}, g_{2}\right)$. Because $\chi\left(\sum\right)=P_{1} \chi\left(M_{1}\right)=$ $P_{2} \chi\left(M_{2}\right), \quad f_{g^{\prime}}^{(1)}$ and $f_{g^{\prime}}^{(2)}$ are covering maps by Lemma 1 . Therefore the induced map $f_{*}: \pi_{1}(M) \rightarrow \pi_{1}(N)$ is injective. By Lemma 3 there exists a branched minimal immersion in the homotopy class of $f$. From the discussion above we know that the branch point cannot occur (since $J\left(f_{g^{\prime}}^{(1)}\right)>0$ ). In summary, we have shown that there exists a unique minimal surface in the homotopy class of $f$ and it is Lagrangian.

Remark. Theorem 1 can also be proved by using Lemma 1 and the result for the simple case $M_{1}=M_{2}$ and $P_{1}=P_{2}=1$. The argument follows from lifting 
the map $f$ into a covering space $\tilde{N}=\sum \times \sum$. One can see that the unique minimal surface in the homtopy class of the diagonal in $\widetilde{N}$, which is a covering of the unique minimal surface in the homotopy class of $f$, is embedded in $\widetilde{N}$.

For other Lagrangian classes which are of the form $\alpha_{1} \times \alpha_{2}, \alpha_{1} \in H_{1}\left(M_{1}\right)$ and $\alpha_{2} \in H_{1}\left(M_{2}\right)$ we have

Theorem 2. Suppose $f=\left(f_{1}, f_{2}\right): T^{2} \rightarrow\left(M_{1}, g_{1}\right) \times\left(M_{2}, g_{2}\right)$, where $T^{2}$ is a torus and $\left[f\left(T^{2}\right)\right]=\alpha_{1} \times \alpha_{2}, \alpha_{i} \in H_{1}\left(M_{i}\right), i=1,2$. Then there exists a unique minimal surface in the homotopy class of $f$ and this minimal surface is Lagrangian.

Proof. Because $\left[f\left(T^{2}\right)\right]=\alpha_{1} \times \alpha_{2}$, we have that $f_{*}: \pi_{1}\left(T^{2}\right) \rightarrow \pi_{1}(N)$ is injective. By Lemma 3 there exists a branched minimal immersion in the homotopy class of $f$. Suppose $\phi\left(T^{2}\right)$ is minimal, $\phi: T^{2} \rightarrow N$ is a conformal harmonic map with respect to the induced metric $g$. One can classify all the harmonic maps from $T^{2} \rightarrow Y$, where $Y$ is a closed Riemannian surface (see [14]). From the conditions we have, we can conclude that $\phi_{1}$ maps $T^{2}$ onto a closed geodesic $C_{1}$ of $\left(M_{1}, g_{1}\right)$ and $\phi_{2}$ maps $T^{2}$ onto a closed geodesic $C_{2}$ of $\left(M_{2}, g_{2}\right)$. Therefore $\phi\left(T^{2}\right)$ is Lagrangian. Let $r_{j}$ be a closed curve in the homotopy class $\left[C_{j}\right]$ with the smallest length. The curve $r_{j}$ will then be a geodesic in $M_{j}$ and there exists a harmonic map $F_{j}$ from $\left(T^{2}, g\right)$ onto $r_{j} \subset M_{j}$. Since $F_{j}$ is homotopic to $f_{j}$, by the uniqueness of harmonic maps in a homotopy class (see [9]) we have $\phi_{j}$ is the composition of a rotation of $r_{j}$ with $F_{j}$. Therefore, $\phi\left(T^{2}\right)=r_{1} \times r_{2}$. Since the images $r_{1}$ and $r_{2}$ are unique, it implies the uniqueness of minimal surfaces in this homotopy class and clearly it is Lagrangian. Moreover, $\operatorname{rank} J\left(\phi_{j}\right)=\operatorname{rank} J\left(F_{j}\right)=1$, but at a branch point $\operatorname{rank} J(\phi)=0$, therefore the minimal surface has no branch point.

About the relations between minimal surfaces and Lagrangian surfaces, we have the following proposition:

Proposition 1. Let $(N, g)$ be a Kähler-Einstein surface with $c_{1}<0$ (not necessarily a product space). Suppose $\beta \in H_{2}(N)$ and there exists an embedded 
totally real surface $\sum_{r}$ of genus $r$ representing $\beta$. Then every connected embedded minimal surface in $\beta$ has genus no less than $r$. The equality holds if and only if the embedded minimal surface is Lagrangian.

Proof. For a real surface $L$ which has isolated complex tangencies in a complex surface, there are relations between the number of complex tangencies and $c_{1}, \chi(L), \chi(\nu)$ where $\chi(\nu)$ is the Euler number for the normal bundle (see [5]). In particular, for a totally real surface which has no complex tangency representing $\beta$, we have

$$
\chi\left(\sum_{r}\right)+\chi(\nu)=0, \text { and } c_{1}(N)(\beta)=0 .
$$

When a surface is embedded, $\chi(\nu)=\left(\beta^{\sharp} \cup \beta^{\sharp}\right)(N)$ where $\beta^{\sharp} \in H^{2}(N ; Z)$ denotes the Poincaré dual of $\beta$. Thus we have

$$
0=(2-2 r)+\left(\beta^{\sharp} \cup \beta^{\sharp}\right)(N)=c_{1}(N)(\beta) .
$$

For a minimal immersion $f(M)$ which is not holomorphic or antiholomorphic in a Kähler surface, J. G. Wolfson proved that its complex and anticomplex points are isolated and each has a negative index. If we denote the numbers of complex points and anticomplex points of $f(M)$ by $P$ and $Q$ respectively, we then have

$$
\begin{aligned}
-P-Q & =\chi(M)+\chi(\nu) \\
Q-P & =c_{1}(N)\left(f_{*}[M]\right) .
\end{aligned}
$$

(See [19], [20], [21] and also compare with [5].) Suppose the genus of $M$ is $k$ and $f(M)$ is embedded. We then get

$$
\begin{aligned}
-P-Q & =(2-2 k)+\left(\beta^{\sharp} \cup \beta^{\sharp}\right)(N) \\
& \leq 0 \\
& =c_{1}(N)(\beta) \\
& =(2-2 r)+\left(\beta^{\sharp} \cup \beta^{\sharp}\right)(N) .
\end{aligned}
$$

It follows that $k \geq r$. The equality holds if and only if $P=Q=0$ which means that $f(M)$ is a totally real surface. When $N$ is a Kähler-Einstein surface of negative scalar curvature, J. G. Wolfson [21] proved that every 
totally real minimal surface in $N$ is a Lagrangian surface. Therefore $f(M)$ is a Lagrangian surface. The case that $f(M)$ is a connected embedded holomorphic or anti holomorphic curve cannot happen in a Lagrangian class. This proves Proposition 1.

Remark 1 . The equation for the embedded totally real surface

$$
0=(2-2 r)+\left(\beta^{\sharp} \cup \beta^{\sharp}\right)(N)=c_{1}(N)(\beta)
$$

depends only on the complex structure. All the arguments go through if the two complex structures are different but have the same first Chern class.

Remark 2. In the proof of Proposition 1, we also show that if there is no restriction on $c_{1}$ of the Kähler-Einstein surface $(N, g)$, then in the conclusion "Lagrangian" is replaced by "totally real". Simlar conclusions hold when $N$ is only a Kähler surface.

\section{REFERENCES}

1. Bers, L., An outline of the theory of pseudo-analytic functions, Bull. Amer. Math. Soc. 42 (1936), 689-692.

2. Bryant, R.L., Minimal Lagrangian submanifolds of Kähler-Einstein manifolds, Lec. Notes in Math. Vol. 1255, 1-12, Berlin Heidelberg New York: Springer 1987.

3. Chen, B.Y., Geometry of submanifolds and its application, Science University of Tokoyo 1981.

4. Eliashberg, Y., private communication.

5. Eliashberg, Y. and Harlamov V., Some remarks on the number of complex points on a real surface in the complex one, Proceedings of Leningrad Int. Topology Conference (1982), 143-148.

6. Eells, J. and Sampson, J.H., Harmonic mappings of Riemannian manifolds, Amer. J. Math. 86 (1964), 109-160.

7. Harvey, R., Spinors and Calibrations, Perspectives in Math. Vol. 9, Academic Press, Inc. 1990.

8. Harvey, R. and Lawson, B., Calibrated geometries, Acta Math. 148 (1982), 48156.

9. Hartman, P., On homotopic harmonic maps, Can. J. Math. 19 (1967), 673-687.

10. Heinz, E., On certain nonlinear elliptic differential equations and univalent mappings, J. Analyse Math. 5 (1956-57), 197-272.

11. Lee, Y.I., Thesis, Stanford University 1992.

12. Micallef, M.J. and Wolfson, J.G., The second variation of area of minimal surfaces in four-manifold, Math. Ann. 295 (1993), 245-267. 
13. Oh, Y.G., Second variation and stabilities of minimal Lagrangian submanifolds in Kähler manifolds, Inv. Math. 101 (1990), 501-519.

14. Sampson, J.H., Some properties and applications of harmonic mappings, Ann. Scient. Éc. Norm. Sup. 11 (1978), 211-228.

15. Schoen, R., The role of harmonic mappings in rigidity and deformation problems, Preprint.

16. Schoen, R. and Yau, S.T., Existence of incompressible minimal surfaces and the topology of three dimensional manifolds with non-negative scalar curvature, Ann. Math. 110 (1979), 127-142.

17. Schoen, R. and Yau, S.T., On univalent harmonic maps between surfaces, Inv. Math. 44 (1978), 265-278.

18. Sacks, J. and Uhlenbeck, K., The existence of minimal immersions of 2-spheres, Ann. of Math. 113 (1981), 1-24.

19. Webster, S., Minimal surfaces in a Kähler surface, J. Diff. Geom. 20 (1984), 463-470.

20. Webster, S., On the relation between Chern and Pontrjagin numbers, Contemporary Math. No. 49 (1986), Amer. Math. Soc., Providence, RI, 135-143.

21. Wolfson, J.G., Minimal surfaces in Kähler surfaces and Ricci curvature, J. Diff. Geom. 29 (1989), 281-294.

National Taiwan University, Taipei, Taiwan.

E-mail address: yilee@math.ntu.edu.tw

RECEIVED APRIL 19, 1994. 